\title{
Efecto alelopático de extractos acuosos foliares de diez ecotipos de trigo (Triticum aestivum L.) sobre Rumex acetosella L.
}

\author{
Allelopathic effect of foliar aqueous extract of ten wild tipe wheat \\ (Triticum aestivum $L$.) on Rumex acetosella $L$.
}

\author{
Claudia Viné L.; Jaime Guerrero C. y Emma Bensch T. ${ }^{*}$
}

\begin{abstract}
RESUMEN
Los objetivos de este estudio fueron determinar el efecto alelopático de extractos acuosos foliares de diez ecotipos de Triticum aestivum L. en dos concentraciones sobre la germinación y crecimiento de la maleza Rumex acetosella L. y clasificarlos según su efectividad. El diseño experimental fue completamente al azar con tres repeticiones. Se evaluó porcentaje de germinación, altura de plántula $(\mathrm{cm})$, longitud de radícula $(\mathrm{cm})$, peso fresco y peso seco de follaje y radical $(\mathrm{g})$. Los resultados fueron evaluados a los 15 días postsiembra y sometidos a un Análisis de Varianza y la comparación de medias con la prueba de significancia mínima (LSD) (P $\leq 0,05)$. La maleza $R$. acetosella manifestó sensibilidad alelopática diferenciada para los diez ecotipos de trigo. Sin embargo, dicha sensibilidad no fue significativa en todos los parámetros evaluados. La mayoría de los ecotipos de trigo ejerció efecto alelopático inhibidor sobre el crecimiento, el que no siempre fue directamente proporcional a la concentración del extracto acuoso evaluado. La germinación de semillas no manifestó efecto alelopático inhibitorio para los ecotipos de trigo. La altura de plántula y largo radical fueron inhibidos por los ecotipos de trigo, al igual que el peso fresco del follaje y peso fresco radical. Con relación a las concentraciones, los resultados más homogéneos, se registraron para largo radical y peso fresco de raíces. Los ecotipos de trigo según su efectividad fueron: Colmillo de perro, Varón y NN7, seguido de NN3, NN8 y NN2; sin registrar inhibición, se ubican Trigo cebada y NN4.
\end{abstract}

Palabras clave: ecotipos de trigo, extractos acuosos, Triticum aestivum, alelopatía.

\begin{abstract}
The objectives of this study was to determine the allelopathic effect of foliar aqueous extracts of ten ecotypes of Triticum aestivum L. in two concentrations about germination and growth of the weed Rumex acetosella L. and classify them under effectiveness. The experimental design was totally randomized design with three replications. We assessed germination percentage, seedling height $(\mathrm{cm})$, root length $(\mathrm{cm})$, fresh weight of foliage and root $(\mathrm{g})$ and dry weight of leaves and roots $(\mathrm{g})$. The results were evaluated 15 days after planting and were subjected to a Variance Analysis and comparison of means with the minimum significance test (LSD) $(P \leq 0,05)$. R. acetosella weed had a differential allelopathic sensitivity to ten wheat ecotypes evaluated. Hawever, this sensitivity was not significant in all parameters. Most wheat ecotypes had an inhibitor allelopathic effect on the growth. Effect was not always directly proportional to the concentration of aqueous extract tested. Seed germination showed no inhibitory allelopathic effect for wheat ecotypes. The growth seedling height and radicular length was sensitive to the inhibitory allelopathic effect just like for the fresh weight of foliage and root fresh weight. Regarding the allelopathic effect of the ecotypes to both concentrations, the more homogeneous results were registered for root length and fresh weight of roots. The ranking of wheat ecotypes according growth inhibition effectiveness to the R. acetosella were: Colmillo de perro, Varón and NN7, followed NN3, NN8 and NN2; unregistered inhibition are located Trigo cabada and NN4.
\end{abstract}

Key words: wild type wheat, aqueous extracts, Triticum aestivum, allelopathy.

\section{Introducción}

El trigo contiene diversos compuestos químicos conocidos por poseer propiedades alelopáticas (Mogensen et al., 2004) y un alto potencial en la supresión de malezas (Bensch et al., 2007, 2009). Tanto la planta como sus residuos pueden ser aprovechados para el manejo de la vegetación no deseada (Wu et al., 2001a). Estos compuestos alelopáticos denominados aleloquímicos se definen como sustancias químicas inhibitorias o fitotóxicas liberadas activamente por las plantas a través de diversas vías (Kogan, 1992; Ormeño, 1997) como son volatilización (principalmente desde hojas),

\footnotetext{
1 Universidad de La Frontera, Facultad de Ciencias Agropecuarias y Forestales.

* Autor para correspondencia: eabensch@ufro.cl
} 
lixiviación (desde follaje o raíces, por medio de lluvia, rocío o niebla), exudación (a través de las raíces) y descomposición de la materia orgánica por microorganismos (Ormeño, 1997; An et al., 1998; Wu et al., 1999; Anaya et al., 2001). Los compuestos con potencial alelopático están presentes en todos los tejidos de las plantas como hojas, tallos, raíces, flores, frutos, semillas y polen (An et al., 1998; Alam et al., 2001), que a través de interacciones alelopáticas pueden inhibir selectivamente la existencia de especies que compiten en sus alrededores, tales como microorganismos del suelo u otras plantas, pudiendo dañar la germinación, crecimiento y desarrollo de otras plantas (Harborne, 1982; Rice, 1984; Evans, 1989). En este sentido la alelopatía puede ser utilizada agronómicamente como en cultivos de supresión o cubiertas alelopáticas vegetales (Eskelsen y Crabtree, 1995; Anaya, 1999; Chou, 1999; Ormeño, 1999), rotaciones alelopáticas o cultivos compañeros (Rice, 1979; Barnes y Putnam, 1986; Wojcik et al., 1990; Pérez y Ormeño, 1991; Ormeño, 1999), incorporación de residuos de cultivos alelopáticos al suelo (Dilday et al., 1994; Chung y Miller, 1995; Anaya, 1999; Ormeño, 1999) y uso de extractos fitotóxicos de plantas alelopáticas (Cheng y Miller, 1995; Chou, 1999; Cheema y Khaliq, 2000).

Altieri (1995), Ormeño (1999), Wu et al., (1999), Olofsdotter et al., (1999), Anaya (1999) y Chou (1999) citando a diversos autores, reportan que son numerosas las especies cultivadas que presentan efectos alelopáticos sobre algunas malezas. Estudios en trigo (Wu et al., 2001a), avena (Fay y Duke, 1977), cebada (Ben-Hammouda y Oueslati, 1999) y arroz (Dilday et al., 1994) reportan variación alelopática entre accesiones sobre la inhibición en el desarrollo de otras especies vegetales.

Debido a la importancia del trigo, se ha evaluado el potencial alelopático diferencial de este cereal, determinando resultados de inhibición positivos (Copaja et al., 1991; Bertholdsson, 2004; Wu et al., 1998, 1999, 2000a, 2000b, 2001b; Zheng et al., 2007; Bensch et al., 2007, 2009).

$R$. acetosella, maleza de nombre común vinagrillo se reproduce por semillas y rizomas (Espinoza, 1996). Según Matthei (1995), es una maleza perenne y dioica ampliamente distribuida, presente en numerosos cultivos, sus extensos rizomas le permiten competir exitosamente con ellos, se encuentra en huertos frutales, hortalizas, cultivos de papas, trigo, avena, remolacha y porotos. En Chile está presente en las regiones segunda, cuarta, quinta, región metropolitana, sexta, séptima, octava, novena, décima, undécima y duodécima (Matthei, 1995). Al tratarse de una maleza presente en huertos frutales de la zona sur, su reproducción por rizomas sumado a su floración y fructificación que comienza en primavera, la transforma en una especie que compite con estos cultivos. Una opción a esto es la utilización de intercultivos, como trigo, para su posterior corte y uso en cultivos de cobertura, ya que además de tener influencia alelopática ayudarían en la incorporación de nutrientes, evitar erosión y pérdida de humedad ente otros. Putnam y Duke (1974) postularon que los tipos silvestres de los cultivos actuales pueden haber poseído una alta actividad alelopática y que este carácter fue reducido o perdido a través de la hibridación y selección para adquirir otras características. Es por ello que se postula que los ecotipos de trigo, al no haber sido seleccionados con un afán de aumentar la producción y al estar más cerca de los progenitores silvestres, podrían tener un potencial alelopático mayor que las variedades cultivadas en el control de malezas.

En este contexto, como hipótesis de trabajo se postula que los extractos acuosos foliares de ecotipos de Triticum aestivum, tienen un efecto alelopático negativo diferencial sobre el crecimiento de la maleza Rumex acetosella. Como objetivos se plantean: determinar el potencial alelopático de extractos acuosos foliares de ecotipos de trigo (Linaza, Castaño colorado, Trigo cebada, Colmillo de perro, Varón, NN2, NN3, NN4, NN7 y NN8) en dos concentraciones, sobre la germinación y crecimiento de $R$. acetosella y clasificarlos según su efectividad.

\section{Materiales y Métodos}

La investigación se realizó en el laboratorio de semillas del Servicio Agrícola y Ganadero, División Temuco, Región de La Araucanía. La germinación de los ecotipos de trigo se realizó en el invernadero del Centro de Agroecología de la Facultad de Ciencias Agropecuarias y Forestales de la Universidad de La Frontera, Temuco. Se evaluó el efecto alelopático de extractos acuosos foliares de ecotipos de trigo, especie dadora, sobre la germinación de semillas y el crecimiento de plántulas de $R$. acetosella, especie receptora. 


\section{Obtención de los extractos acuosos}

Las semillas de los ecotipos de trigo fueron sembradas en macetas de 1,0 litro, usando como sustrato suelo trumao. Se utilizó un total de 1000 semillas por ecotipo, divididas en 10 macetas. Fueron regadas con agua potable según necesidad, manteniendo el suelo a Capacidad de Campo, permaneciendo en invernadero por 17 días. Posteriormente se extrajo la parte aérea de las plántulas, las que se depositaron en bolsas de papel, clasificadas por ecotipo.

De acuerdo con lo planteado por LaynezGarsaball y Méndez-Natera (2006), las plántulas de trigo fueron extraídas de las macetas a los 17 días post siembra. Posteriormente se secaron a temperatura ambiente por $24 \mathrm{~h}$ y luego en estufa a $50{ }^{\circ} \mathrm{C}$ por $72 \mathrm{~h}$. Una vez seca, las plántulas fueron cortadas en trozos no mayores a $3 \mathrm{~cm}$ y licuados en agua sin pulverizar (10 s. aprox.). La preparación se dejó en reposo por $48 \mathrm{~h}$ en recipientes de vidrio de $500 \mathrm{cc}$ tapados con papel de polipropileno. Luego se separó el líquido de la parte sólida, a través de un proceso de filtrado, utilizando bomba de vacío (papel filtro Whatman 1), para obtener un extracto al 2,0\% p/v. A partir de esto se obtuvieron por dilución con agua destilada extractos al 1,0 y $2,0 \% \mathrm{p} / \mathrm{v}$. Como tratamiento control se empleó agua destilada.

\section{Siembra}

Las semillas de vinagrillo fueron refrigeradas por 30 días a $4{ }^{\circ} \mathrm{C}$ en primera instancia; con la finalidad de romper su latencia, para la germinación se utilizaron placas petri de $90 \times 15 \mathrm{~mm}$, desinfectadas con alcohol. Como sustrato se utilizaron cuatro capas de papel absorbente, humedecidas con agua destilada, con 25 semillas por placa y tres repeticiones por tratamiento. Se dispusieron 24 placas por bandeja plástica cubiertas con bolsas de polietileno; se mantuvieron en cámara de temperatura alterna $\left(20-30{ }^{\circ} \mathrm{C}\right)$ por seis días. Una vez germinadas se trasladaron a la cámara de cultivo con temperatura constante de $20^{\circ} \mathrm{C}$, donde permanecieron por otros nueve días. Se realizaron tres riegos con 1,0 $\mathrm{ml}$ de cada extracto desde la germinación hasta completar los 15 días; se mantuvo mayor humedad en el sustrato gracias a las bolsas de polietileno, que impedían la evaporación.

\section{Diseño experimental}

Se empleó un diseño completamente al azar, con once tratamientos y tres repeticiones, considerando como tratamientos los extractos acuosos de los diez ecotipos de trigo más agua destilada como control. El estudio fue evaluado en las concentraciones 1,0 y $2,0 \% \mathrm{p} / \mathrm{v}$ y se consideró como unidad experimental la placa petri.

\section{Variables medidas}

Las evaluaciones en la especie receptora R. acetosella consideraron: A los seis días post siembra el porcentaje de germinación y a los 15 días post siembra la altura de plántula $(\mathrm{cm})$, longitud de radícula $(\mathrm{cm})$, peso fresco y peso seco de follaje y radical (g). Inderjit y Weston (2000) señalan que en el estudio de alelopatía se debería enfatizar en la actividad alelopática durante el estado inicial de crecimiento, ya que la interacción entre cultivo y maleza, es crítica en este período.

\section{Análisis estadístico}

Previa comprobación de los supuestos de homogeneidad de varianza y distribución normal, los datos paramétricos fueron avaluados mediante análisis estadístico de varianza y los promedios de las medias a través de prueba de significancia mínima (LSD) $(\mathrm{P} \leq 0,05)$. Todos los análisis fueron realizados mediante el programa Statgraphics Plus 5.1; los gráficos se realizaron en Microsoft Excel.

\section{Resultados y Discusión}

El estudio estableció que la maleza $R$. acetosella tuvo sensibilidad alelopática diferencial frente a los diez ecotipos de trigo evaluados, donde la mayoría ejerció efecto inhibidor. Las diferencias observadas entre ecotipos podría explicarse en parte debido a la sensibilidad diferencial de la maleza a los aleloquímicos de los ecotipos de trigo, lo cual está asociado a variados factores basados en una interacción esencialmente de tipo bioquímico y enzimático de los extractos de la especies alelopáticas (Wu et al., 2000b). Los resultados concuerdan con lo reportado en la literatura, donde se señala que tanto la concentración como la naturaleza de los compuestos alelopáticos 
influyen en la inhibición de especies (Einhelling, 1995; An et al., 1998; Wu et al., 2000a, 2000b). Dentro de los mismos ecotipos hubo respuestas diferentes según la concentración de extracto acuoso. Según Wu et al. (1999), la variabilidad genética de la característica alelopática es amplia dentro de las especies cultivadas, se agrega lo planteado por Tang (1986), que considera que las condiciones ambientales externas tienen una gran influencia en la expresión alelopática, además Chou (1999) menciona al respecto que la cantidad de aleloquímicos liberados por una planta es significativamente mayor cuando esta crece en situación adversa, en relación a lo producido por una planta que se ha desarrollado bajo condiciones de laboratorio. Los efectos alelopáticos no siempre poseen una explicación única, puesto que corresponde a un proceso complejo y muy dinámico, en que a veces no es posible aislar los factores que están interviniendo en este proceso natural de interacciones.

\section{Efecto de los extractos sobre la germinación}

Se detectaron diferencias significativas en el ecotipo Colmillo de perro, el cual presentó efecto alelopático inhibidor $(7,33 \%)$ en la concentración de $1,0 \%$, los nueve ecotipos restantes no se diferenciaron significativamente del testigo. En la concentración de $2,0 \%$ no se presentaron diferencias significativas entre los tratamientos, ni entre estos y el testigo (Cuadro 1 y Figura 1). Los resultados obtenidos en este parámetro pueden estar en función de la sensibilidad alelopática de la especie receptora a aleloquímicos de trigo, de lo cual depende el efecto inhibitorio provocado por los extractos, lo que está relacionado con el tipo y concentración de aleloquímicos en el extracto o de la combinación de estos, que estarían correlacionados, dependiendo en cada caso con el genotipo de la especie dadora (Einhellig, 1995; An et al., 1998; Wu et al., 2000a, 2000b, 2001b). Para el caso de trigo existe evidencia de diferente potencial alelopático entre variedades para el control de malezas, destacando un estudio con exudados de raíces de plántulas de 92 variedades de trigo y su efecto sobre ballica (Lolium rigidum) (Wu et al., 2000a). También se han observado diferencias entre el extracto acuoso de rastrojo de trigo de tres variedades de trigo sobre la germinación en raps (Bruce y Christen, 2001). Wu et al (2001a) afirman que los aleloquímicos son distribuidos diferencialmente en brotes y raíces de plántulas de trigo, presentándose en mayor cantidad en las raíces. Sin embargo, no es claro que las altas cantidades de aleloquímicos encontrados en la raíz sean derivadas de su directa biosíntesis, de la traslocación de estos desde los brotes o de ambos.

Cuadro 1. Germinación (\%) de semillas de R. acetosella, según concentración al $1,0 \%$ y $2,0 \%$ p/v de extractos acuosos foliares de ecotipos de trigo.

\begin{tabular}{|c|c|c|}
\hline \multirow{2}{*}{ Cultivar } & \multicolumn{2}{|c|}{ Concentración (p/v) } \\
\hline & $1 \%$ & $2 \%$ \\
\hline NN7 & $90,67 \mathrm{ab}$ & 97,33 \\
\hline Varón & $94,67 \mathrm{abc}$ & 97,33 \\
\hline Colmillo de perro & 89,33 a & 97,33 \\
\hline NN3 & $97,33 \mathrm{c}$ & 100,00 \\
\hline Linaza & $96,00 \mathrm{bc}$ & 96,00 \\
\hline Castaño colorado & $100,00 \mathrm{c}$ & 96,00 \\
\hline NN8 & $97,33 \mathrm{c}$ & 100,00 \\
\hline Trigo cebada & $100,00 \mathrm{c}$ & 97,33 \\
\hline NN4 & $98,67 \mathrm{c}$ & 97,33 \\
\hline NN2 & $98,67 \mathrm{c}$ & 100,00 n.s. \\
\hline Promedio & 96,27 & 97,87 \\
\hline
\end{tabular}

Letras distintas en columna indica diferencia significativa según $\operatorname{LSD}(\mathrm{P} \leq 0.05)$.

n.s. $=$ no significativo.

Germinación promedio del testigo: 96,4\% promedio de 30 evaluaciones de $R$. acetosella.

\section{Efecto de los extractos sobre altura de plántula y largo radical}

Para altura de plántula se evidenciaron diferencias significativas entre los ecotipos, no así, entre estos y el testigo en la concentración de 1,0\%. Con 2,0\%, se obtuvo significancia entre los ecotipos y entre estos y el testigo, dos ecotipos manifestaron efecto alelopático, NN8 $(15,37 \%)$ efecto inhibidor y NN3 $(+21,63 \%)$ efecto potenciador, el resto no logró relevancia para la maleza en estudio (Cuadro 2 y Figura 2). Para el largo radical se registraron diferencias significativas en ambas concentraciones entre tratamientos y entre estos y el testigo. Tres ecotipos tuvieron respuesta alelopática inhibitoria en la concentración de $1,0 \%$ sin diferencias significativas entre ellos, NN7 (28,74\%), Colmillo de perro $(28,10 \%)$ y Varón $(21,80 \%)$, los ecotipos restantes no presentaron diferencias con el testigo. En la concentración de 2,0\% seis ecotipos tuvieron potencial alelopático inhibidor sobre $R$. acetosella, NN7 (32,62\%), Castaño colorado (29,55\%), NN3 $(27,61 \%)$, Varón $(22,44 \%)$, Linaza $(20,18 \%)$, 


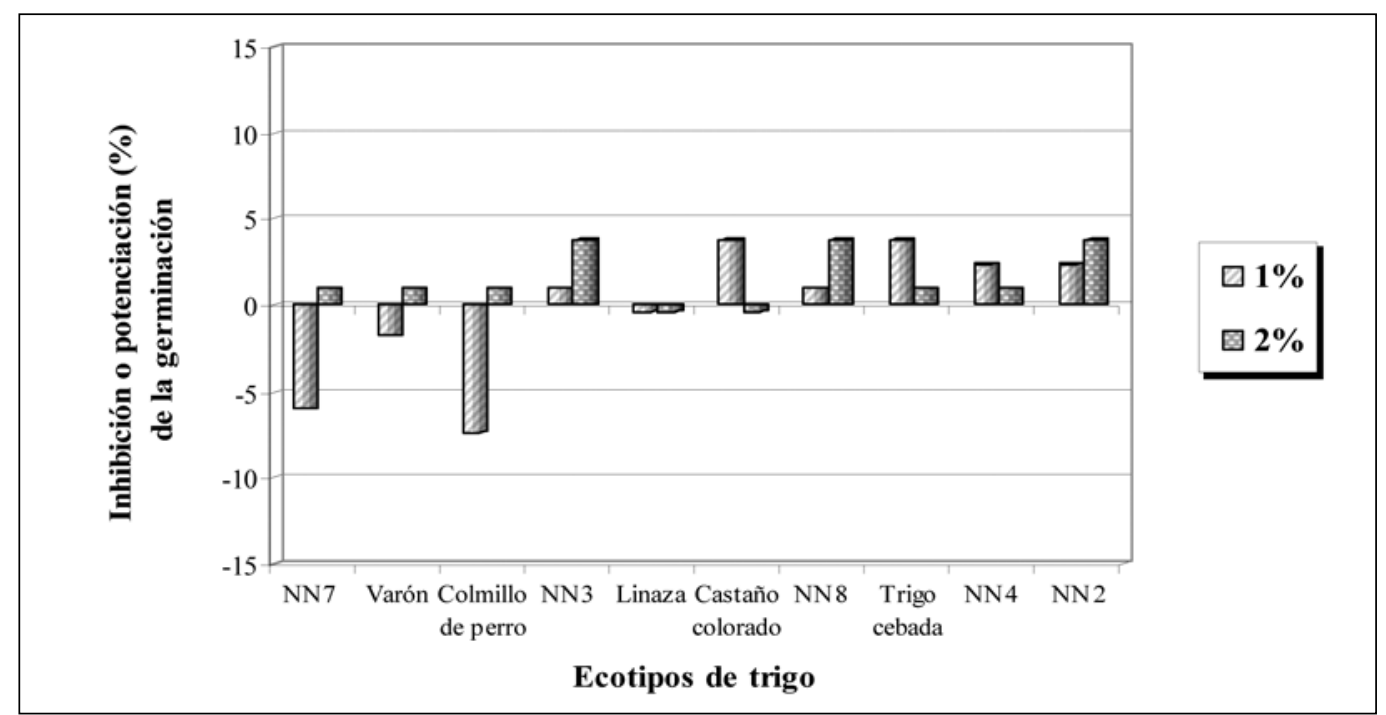

Figura 1. Inhibición o potenciación (\%) de la germinación de semillas de R. acetosella, según concentración al 1,0\% y 2,0\%p/v de extractos acuosos foliares de ecotipos de trigo, respecto del testigo.

Colmillo de perro (18,89\%). NN8, Trigo cebada, NN4 y NN2 se comportaron significativamente equivalentes al testigo (Figura 3 ).

Entre el índice de crecimiento de plántula y el índice del crecimiento radical en su mayoría, no se apreciaron coincidencias respecto de la interacción alelopática sobre la maleza evaluada. Se estableció en ambos casos que la concentración mas alta fue la más influyente. Anaya y CruzOrtega (2001) sugieren que los aleloquímicos poseen diversos sitios y mecanismos de acción biológica sobre el organismo receptor, lo que se traduce en mayor o menor crecimiento y desarrollo. El parámetro de crecimiento radical presentó mayor uniformidad entre los resultados de ambas concentraciones, NN7 registró el mayor grado de potencial alelopático inhibitorio en ambas concentraciones. El resultado inhibidor en 1,0\% para Colmillo de perro concuerda con el obtenido en inhibición de la germinación.

Cuadro 2. Altura de plántula $(\mathrm{cm})$ y largo de raíz $(\mathrm{cm})$ de $R$. acetosella, según concentración al 1,0\% y $2,0 \% \mathrm{p} / \mathrm{v}$ de extractos acuosos foliares de ecotipos de trigo.

\begin{tabular}{lccccc}
\hline \multirow{2}{*}{ Cultivar } & \multicolumn{5}{c}{ Concentración (p/v) } \\
\cline { 2 - 3 } \cline { 2 - 3 } \cline { 5 - 6 } & \multicolumn{2}{c}{ Altura plántula $(\mathrm{cm})$} & & \multicolumn{2}{c}{ Largo radícula $(\mathrm{cm})$} \\
\cline { 2 - 3 } \cline { 5 - 6 } NN7 & $1,73 \mathrm{bc}$ & $1,81 \mathrm{c}$ & & $1,47 \mathrm{a}$ & $1,39 \mathrm{a}$ \\
Varón & $1,78 \mathrm{c}$ & $1,78 \mathrm{bc}$ & & $1,61 \mathrm{ab}$ & $1,6 \mathrm{a}$ \\
Colmillo de perro & $1,76 \mathrm{c}$ & $1,70 \mathrm{bc}$ & & $1,48 \mathrm{a}$ & $1,67 \mathrm{a}$ \\
NN3 & $1,79 \mathrm{c}$ & $2,03 \mathrm{~d}$ & & $1,77 \mathrm{abc}$ & $1,49 \mathrm{a}$ \\
Linaza & $1,67 \mathrm{abc}$ & $1,59 \mathrm{ab}$ & & $1,82 \mathrm{abc}$ & $1,65 \mathrm{a}$ \\
Castaño colorado & $1,66 \mathrm{abc}$ & $1,62 \mathrm{bc}$ & & $1,90 \mathrm{bcd}$ & $1,45 \mathrm{a}$ \\
NN8 & $1,56 \mathrm{ab}$ & $1,41 \mathrm{a}$ & & $2,26 \mathrm{de}$ & $2,23 \mathrm{~b}$ \\
Trigo cebada & $1,75 \mathrm{bc}$ & $1,64 \mathrm{bc}$ & & $2,29 \mathrm{de}$ & $2,14 \mathrm{~b}$ \\
NN4 & $1,82 \mathrm{c}$ & $1,63 \mathrm{bc}$ & & $2,09 \mathrm{cde}$ & $2,20 \mathrm{~b}$ \\
NN2 & $1,49 \mathrm{a}$ & $1,62 \mathrm{bc}$ & & $2,31 \mathrm{e}$ & $2,40 \mathrm{~b}$ \\
Promedio & 1,70 & 1,68 & & 1,9 & 1,82 \\
\hline
\end{tabular}

Letras distintas en columna indica diferencia significativa según LSD $(\mathrm{P} \leq 0.05)$.

Altura de plántula promedio del testigo: $1,67 \mathrm{~cm}$, promedio de 300 evaluaciones de $R$. acetosella. Largo radicular promedio del testigo: $2,06 \mathrm{~cm}$, promedio de 300 evaluaciones de $R$. acetosella. 


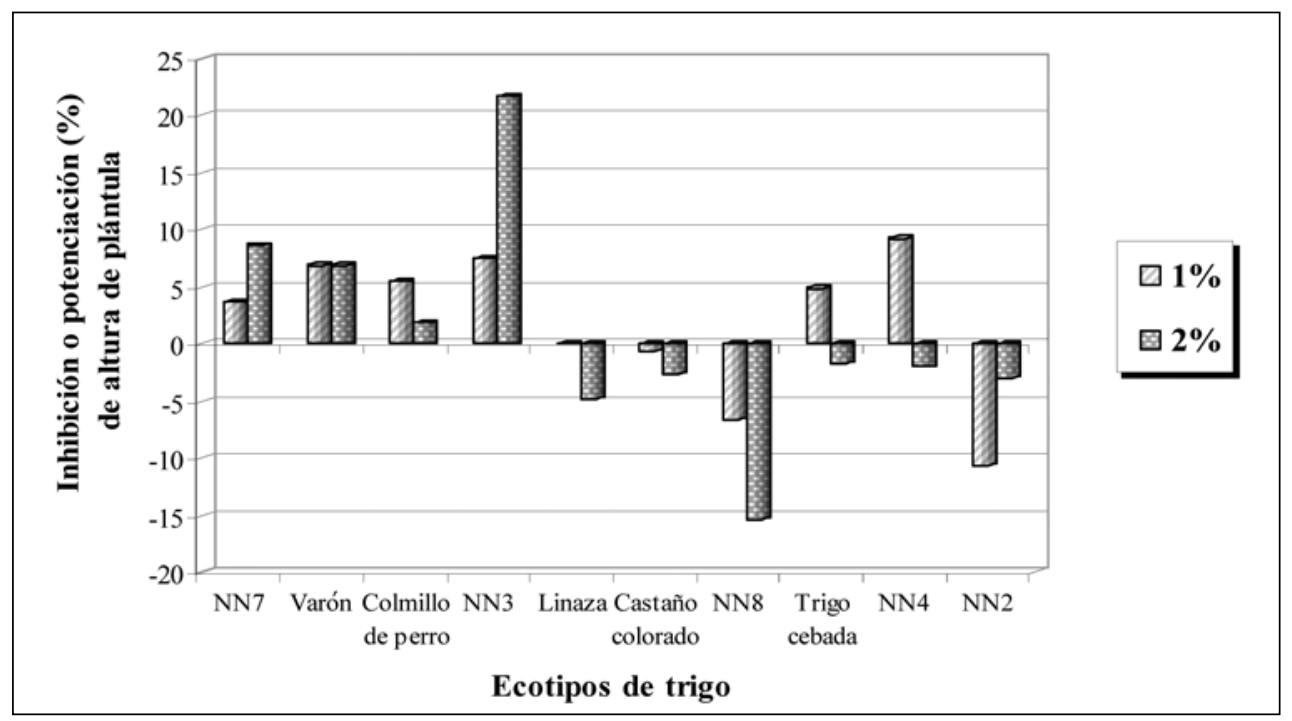

Figura 2. Inhibición o potenciación (\%) de altura de plántula de R. acetosella, según concentración al 1,0\% y 2,0\% p/v de extractos acuosos foliares de ecotipos de trigo.

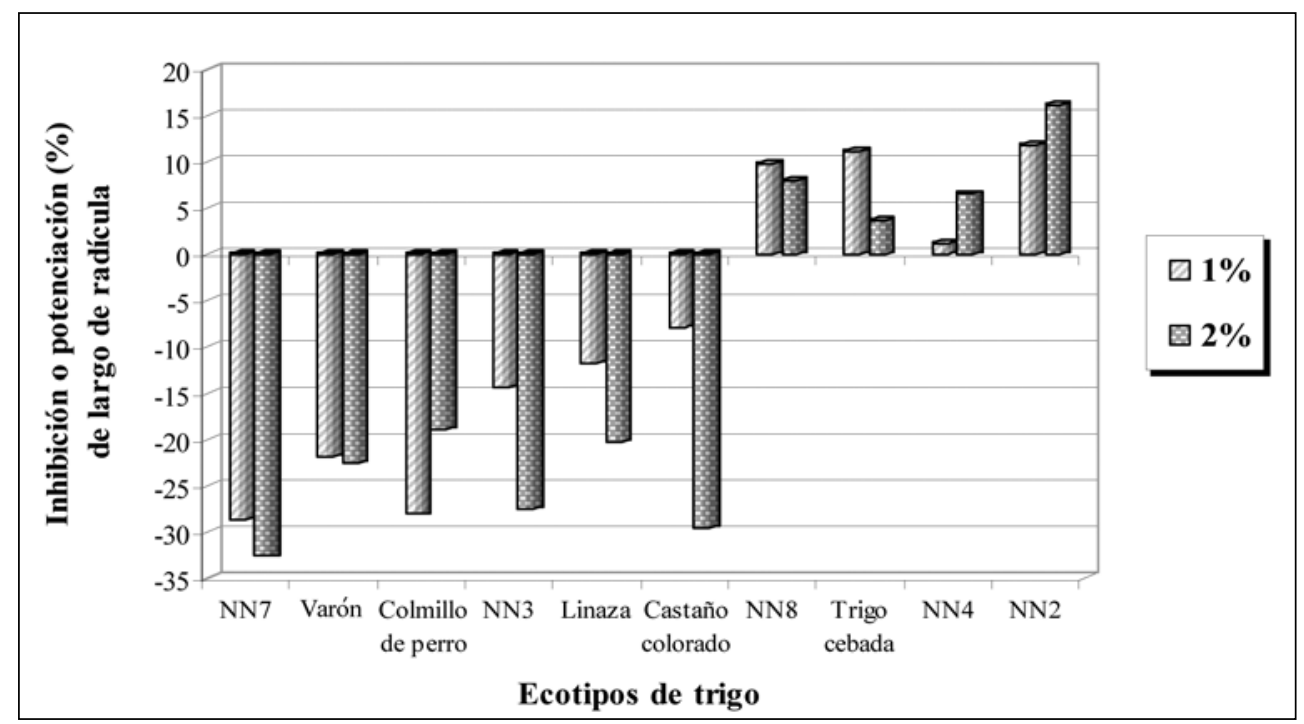

Figura 3. Inhibición o potenciación (\%) de largo de raíz de R. acetosella, según concentración al 1,0\% y 2,0\% p/v de extractos acuosos foliares de ecotipos de trigo.

\section{Efecto de los extractos sobre peso fresco $y$ peso seco de follaje}

Se presentaron diferencias significativas entre los tratamientos y entre estos y el testigo, tanto para el peso fresco como seco en ambas concentraciones. Junto con un efecto inhibidor se detectó potenciación en el parámetro de peso fresco, como se aprecia para la concentración de $1,0 \%$ con Linaza $(+6,41 \%)$, Castaño colorado $(+7,69 \%)$,
NN4 $(+10,13 \%)$, NN3 $(+10,26 \%)$ y Trigo cebada $(+16,79 \%)$, los ecotipos que ejercieron efecto inhibidor fueron NN7 (7,69\%), NN2 $(4,87 \%)$, Colmillo de perro $(3,85 \%)$ y NN8 $(1,79 \%)$. Con la concentración de $2,0 \%$ se detectó potenciación en NN2 $(+3,21 \%)$, NN7 y Linaza $(+3,85 \%)$, Trigo cebada $(+4,87 \%)$ y NN4 $(+5,64 \%)$, el efecto inhibitorio lo registraron Colmillo de perro $(7,69 \%)$, NN8 (3,97\%), Varón y Castaño colorado $(3,85 \%)$ y NN3 $(1,28 \%)$ (Cuadro 3 y Figura 4). El parámetro 
Cuadro 3. Peso fresco y peso seco (g) de parte aérea de R. acetosella, según concentración al 1,0\% y $2,0 \% \mathrm{p} / \mathrm{v}$ de extractos acuosos foliares de ecotipos de trigo.

\begin{tabular}{|c|c|c|c|c|}
\hline \multirow{4}{*}{ Cultivar } & \multicolumn{4}{|c|}{ Peso follaje (g) } \\
\hline & \multicolumn{4}{|c|}{ Concentración $(\mathrm{p} / \mathrm{v})$} \\
\hline & \multicolumn{2}{|c|}{$1 \%$} & \multicolumn{2}{|c|}{$2 \%$} \\
\hline & fresco & seco & fresco & seco \\
\hline NN7 & $0,072 \mathrm{a}$ & $0,005 \mathrm{~b}$ & $0,081 \mathrm{f}$ & $0,005 \mathrm{~b}$ \\
\hline Varón & $0,078 \mathrm{e}$ & $0,005 \mathrm{~b}$ & $0,075 \mathrm{c}$ & $0,005 \mathrm{~b}$ \\
\hline Colmillo de perro & $0,075 \mathrm{c}$ & $0,004 \mathrm{a}$ & $0,072 \mathrm{a}$ & $0,004 \mathrm{a}$ \\
\hline NN3 & $0,086 \mathrm{i}$ & $0,004 \mathrm{a}$ & $0,077 \mathrm{~d}$ & $0,004 \mathrm{a}$ \\
\hline Linaza & $0,083 \mathrm{f}$ & $0,004 \mathrm{a}$ & $0,081 \mathrm{f}$ & $0,004 \mathrm{a}$ \\
\hline Castaño colorado & $0,084 \mathrm{~g}$ & $0,005 \mathrm{~b}$ & $0,075 \mathrm{c}$ & $0,005 \mathrm{~b}$ \\
\hline NN8 & $0,077 \mathrm{~d}$ & $0,004 \mathrm{a}$ & $0,075 \mathrm{~b}$ & $0,005 \mathrm{~b}$ \\
\hline Trigo cebada & $0,091 \mathrm{j}$ & $0,005 \mathrm{~b}$ & $0,082 \mathrm{~g}$ & $0,005 \mathrm{~b}$ \\
\hline NN4 & $0,086 \mathrm{~h}$ & $0,004 \mathrm{a}$ & $0,082 \mathrm{~h}$ & $0,004 \mathrm{a}$ \\
\hline $\mathrm{NN} 2$ & $0,074 \mathrm{~b}$ & $0,004 \mathrm{a}$ & $0,081 \mathrm{e}$ & $0,004 \mathrm{a}$ \\
\hline Promedio & 0,081 & 0,004 & 0,078 & 0,005 \\
\hline
\end{tabular}

Letras distintas en columna indica diferencia significativa según LSD $(\mathrm{P} \leq 0.05)$.

Peso fresco follaje promedio del testigo: $0,078 \mathrm{~g}$, promedio de 10 evaluaciones de $R$. acetosella. Peso seco follaje promedio del testigo: 0,004 g, promedio de 10 evaluaciones de $R$. acetosella.

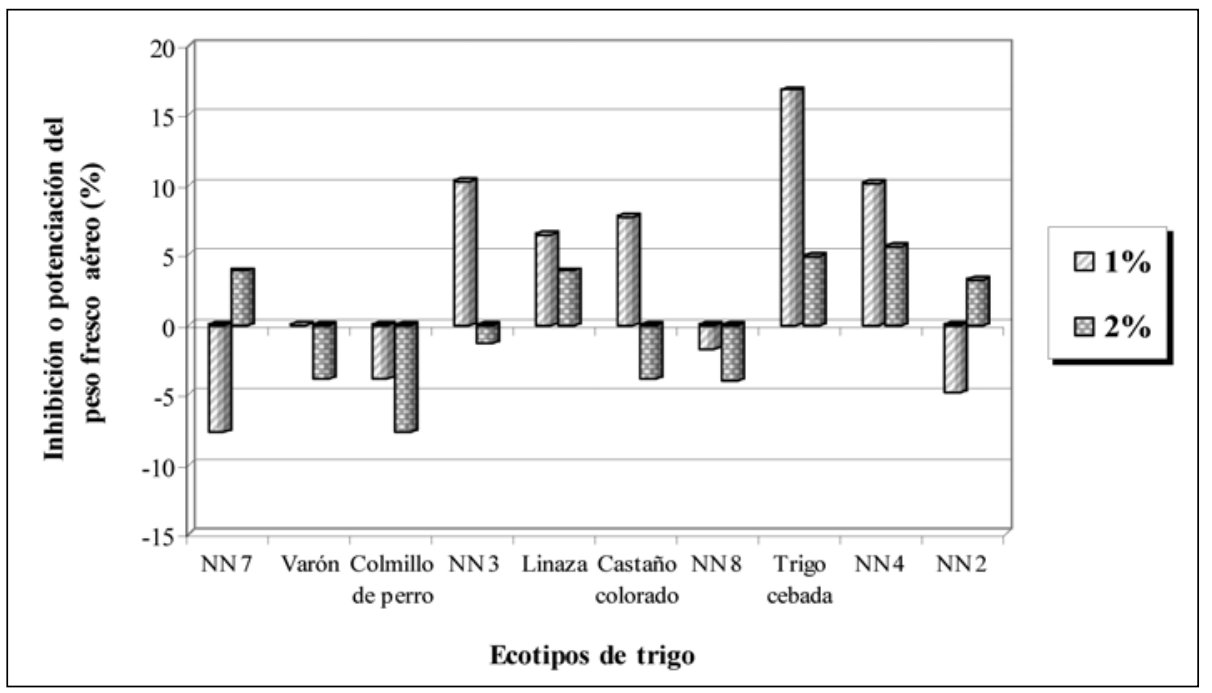

Figura 4. Inhibición o potenciación (\%) del peso fresco del follaje de $R$. acetosella, según concentración al 1,0\% y 2,0\% p/v de extractos acuosos foliares de ecotipos de trigo, con respecto al testigo.

de peso seco solo registró efecto potenciador $(25 \%$ sobre el testigo en todos los casos) con los ecotipos NN7, Varón, Castaño colorado y Trigo cebada, en ambas concentraciones y NN8 en la concentración de 2,0\%. La estimulación del crecimiento de la especie receptora es indicada en la literatura como un hecho relativamente habitual en varias especies cultivadas (Chung et al., (2001) con arroz y Wu et al. $(1998,2000 \mathrm{a}, 2001 \mathrm{a})$ con trigo. Para que se produzcan los efectos alelopáticos ya sean de carácter positivo o negativo, directos o indirectos, la concentración de las sustancias aleloquímicas es de gran importancia y a esto se une el papel de la sensibilidad de la especie receptora (Bowen, 1991). La respuesta inhibitoria de algunos extractos coincide con los resultados obtenidos en el largo radical y germinación en la concentración de 1,0\% con NN7, y Colmillo de perro, los que también concuerda con peso fresco del follaje para 2,0\% Varón, Colmillo de perro, NN3 y Castaño colorado. 


\section{Efecto de los extractos sobre peso fresco y peso seco radical}

Se establecieron diferencias significativas entre los tratamientos y entre estos y el testigo, para la variable de peso fresco en ambas concentraciones. Respecto del peso seco radical, en la concentración de $1,0 \%$ todos los tratamientos obtuvieron idénticos resultados al igual que el testigo, para la concentración de 2,0\% existieron diferencias significativas con respecto al testigo. Se produjo efecto inhibidor como potenciador del peso fresco radical, en la concentración de $1,0 \%$ están NN2 $(+28,18 \%)$, Trigo cebada $(+40,0 \%), \mathrm{NN} 8(+40,91 \%)$ y NN4 $(+44,55 \%)$, ejercieron inhibición los ecotipos NN3 y Castaño colorado $(54,55 \%)$, Varón $(45,45 \%)$, Colmillo de perro y Linaza $(36,36 \%)$ y NN7 $(27,27 \%)$. Para la concentración de $2,0 \%$ potenciaron $\mathrm{NN} 8(+21,82 \%)$, NN4 $(+34,55 \%)$, Trigo cebada $(+59,09 \%)$ y NN2 $(+70,91 \%)$, con efecto inhibidor están Varón, Colmillo de perro y Castaño colorado (54,55\%), NN3 (36,36\%), NN7 $(18,18 \%)$ y Linaza $(9,09 \%)$ (Cuadro 4 y Figura 5). El parámetro de peso seco únicamente registró efecto potenciador en dos ecotipos (50\% sobre el testigo en ambos casos) con Trigo cebada y NN2. Los resultados del peso fresco radical en ambas concentraciones presentaron mayor homogeneidad que para el caso de peso fresco de follaje. Además se destaca similitud en el efecto de inhibición o estimulación sobre $R$. acetosella, con respecto al crecimiento radical. A diferencia de los resultados de este último parámetro, los ecotipos que presentaron el mayor grado de inhibición fueron Castaño colorado en ambas concentraciones, Varón y Colmillo de perro con $2,0 \%$ y NN3 con 1,0\%. Los resultados podrían explicarse de acuerdo con lo que plantean Putnam y Duke (1974), quienes indican que cultivares más antiguos estarían más próximos genéticamente a sus progenitores silvestres; por tanto, su potencial alelopático sería mayor. En cuanto a la naturaleza química de los extractos de los ecotipos de trigo evaluados, Mathiasen et al. (2004) señalan que el compuesto químico identificado como más activo en trigo es el ácido hidroxámico 2,4-Dihidroxi7-metoxi-1,4-benzoxazin-3-ona (DIMBOA). Sin embargo, la presencia de este compuesto ha resultado ser altamente dependiente de la variedad del mismo, lo cual indica que está determinado por factores genéticos (Wu et al., 2007). Lo señalado anteriormente podría explicar las diferencias encontradas entre los ecotipos de trigo evaluados en este estudio. Sin embargo, para determinar con exactitud los compuestos químicos involucrados en la inhibición de la maleza evaluada, es necesario aislar e identificar los compuestos químicos presentes en los extractos obtenidos, con lo cual se podría determinar si efectivamente la diferencia manifestada por los ecotipos se debe a la presencia de DIMBOA o a otro compuesto.

Cuadro 4. Peso fresco y peso seco (g) de la raíz de $R$. acetosella, según concentración al 1,0\% y $2,0 \% \mathrm{p} / \mathrm{v}$ de extractos acuosos foliares de ecotipos de trigo.

\begin{tabular}{|c|c|c|c|c|}
\hline \multirow{4}{*}{ Cultivar } & \multicolumn{4}{|c|}{ Peso raíz (g) } \\
\hline & \multicolumn{4}{|c|}{ Concentración $(\mathrm{p} / \mathrm{v})$} \\
\hline & \multicolumn{2}{|c|}{$1 \%$} & \multicolumn{2}{|c|}{$2 \%$} \\
\hline & fresco & seco & fresco & seco \\
\hline NN7 & $0,008 \mathrm{~d}$ & 0,002 & $0,009 \mathrm{c}$ & $0,002 \mathrm{a}$ \\
\hline Varón & $0,006 \mathrm{~b}$ & 0,002 & $0,005 \mathrm{a}$ & $0,002 \mathrm{a}$ \\
\hline Colmillo de perro & $0,007 \mathrm{c}$ & 0,002 & $0,005 \mathrm{a}$ & $0,002 \mathrm{a}$ \\
\hline NN3 & $0,005 \mathrm{a}$ & 0,002 & $0,007 \mathrm{~b}$ & $0,002 \mathrm{a}$ \\
\hline Linaza & $0,007 \mathrm{c}$ & 0,002 & $0,010 \mathrm{~d}$ & $0,002 \mathrm{a}$ \\
\hline Castaño colorado & $0,005 \mathrm{a}$ & 0,002 & $0,005 \mathrm{a}$ & $0,002 \mathrm{a}$ \\
\hline NN8 & $0,016 \mathrm{~g}$ & 0,002 & $0,013 \mathrm{e}$ & $0,002 \mathrm{a}$ \\
\hline Trigo cebada & $0,015 \mathrm{f}$ & 0,002 & $0,018 \mathrm{~g}$ & $0,003 \mathrm{~b}$ \\
\hline NN4 & $0,016 \mathrm{~h}$ & 0,002 & $0,015 \mathrm{f}$ & $0,002 \mathrm{a}$ \\
\hline $\mathrm{NN} 2$ & $0,014 \mathrm{e}$ & 0,002 n.s. & $0,019 \mathrm{~h}$ & $0,003 \mathrm{~b}$ \\
\hline Promedio & 0,010 & 0,002 & 0,011 & 0,002 \\
\hline
\end{tabular}

Letras distintas en columna indica diferencia significativa según LSD $(\mathrm{P} \leq 0.05)$.

n.s. $=$ no significativo.

Peso fresco radicular promedio del testigo: $0,011 \mathrm{~g}$, promedio de 10 evaluaciones de $R$. acetosella.

Peso seco radicular promedio del testigo: $0,002 \mathrm{~g}$, promedio de 10 evaluaciones de $R$. acetosella. 


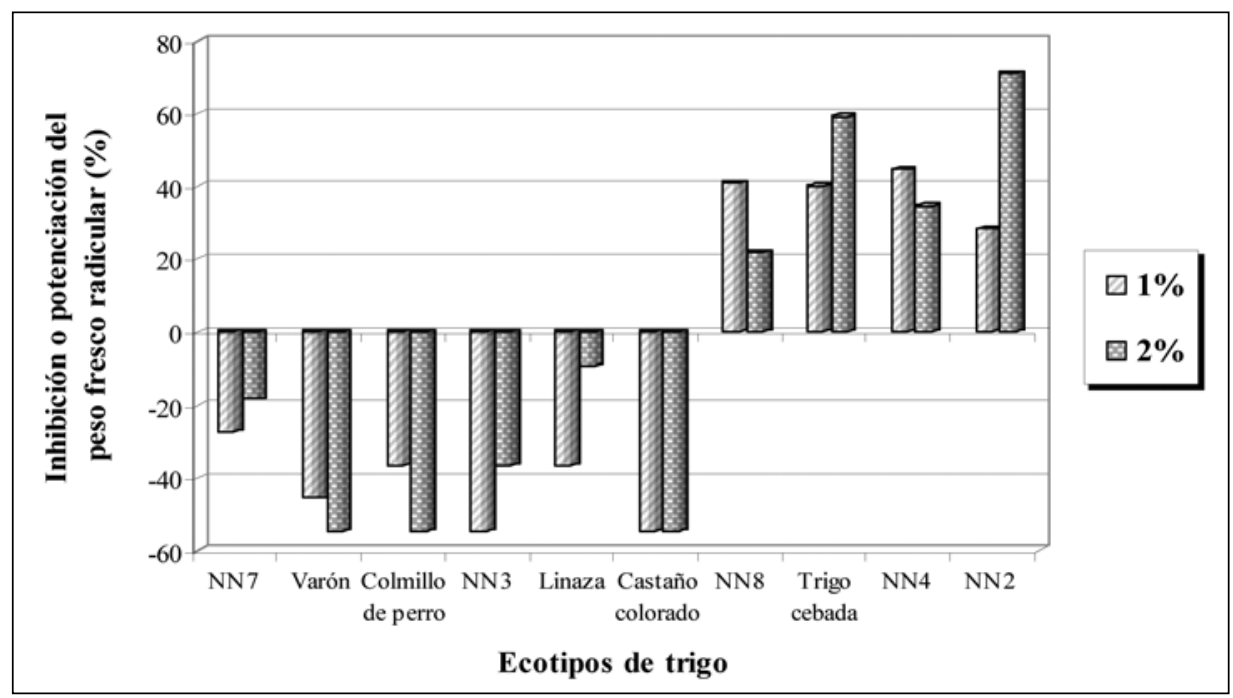

Figura 5. Inhibición o potenciación (\%) del peso fresco radical de $R$. acetosella, según concentración al 1,0\% y 2,0\% p/v de extractos acuosos foliares de ecotipos de trigo, respecto del testigo.

\section{Conclusiones}

1. La maleza Rumex acetosella manifestó sensibilidad alelopática diferenciada para los diez ecotipos de trigo. Sin embargo, dicha sensibilidad no fue significativa en todos los parámetros evaluados.

2. La mayoría de los ecotipos de trigo ejerció efecto alelopático inhibidor sobre el crecimiento de Rumex acetosella. Efecto que no siempre fue directamente proporcional a la concentración del extracto acuoso evaluado.

3. La germinación de semillas de Rumex acetosella no manifestó efecto alelopático inhibitorio para los ecotipos de trigo. El crecimiento de altura foliar y largo radical fue sensible al efecto alelopático inhibitorio al igual que el peso fresco aéreo y peso fresco radical.

4. Con relación al efecto alelopático de los ecotipos en ambas concentraciones, los resultados más homogéneos se registraron para largo radical y peso fresco de raíces.

5. La jerarquización de los ecotipos de trigo según su efectividad sobre la inhibición del crecimiento de Rumex acetosella fue la siguiente: Colmillo de perro, Varón y NN7, seguido de NN3, NN8 y NN2; sin registrar inhibición se ubican Trigo cebada y NN4.

\section{Literatura Citada}

Alam, S.; Ala, S.; Azmi, M.; Ansari, R.

2001. Allelopathy and its role in agriculture. Journal of Biological Sciences. 1: 308-315.

Altieri, M.

1995. Agroecología. Bases Científicas para una Agricultura Sustentable. Golden Horn Press, Berkeley. 281 p.

An, M.; Pratley, J.; Haig, T.

1998. Allelopathy: from concept to reality. Proceedings of the $9^{\text {th }}$ Australian Agronomy Conference. Wagga wagga, Australia. pp. 56-62.

Anaya, A.

1999. Allelopathy as tool in the management of biotic resources in Agroecosystems. Critical Reviews in Plant Sciences 18: 697-739.
Anaya, A.; Cruz-Ortega, R.

2001. La alelopatía: algunos estudios de caso y posibles aplicaciones. In: Anaya, A., Espinosa-García, F. y CruzOrtega, R. (eds.). Relaciones químicas entre organismos: aspectos básicos y perspectivas de su aplicación. Instituto de Ecología, UNAM. Plaza y Valdés, S.A. de C.V. México. pp. 33-68.

Anaya, A.; Espinosa-García, F.; Cruz-Ortega, R.

2001. Relaciones químicas entre organismos: aspectos básicos y perspectivas de su aplicación. Instituto de Ecología, UNAM. Plaza y Valdés, S.A. de C.V. México. 733 p.

Barnes, J.; Putnam, A.

1986. Evidence for allelopathy by residues and aquous extract of rye (Secale cereale L.). Weed Science 34: 384-390. 
Ben-Hammouda, M.; Oueslati, O.

1999. A germination bioassay to test the allelopathic potential of barley. RACHIS Newsletter, 18: 51-54.

Bensch, E.; Schalchli, H.; Fuentes, R.; Seemann, P.; Jobet, C. 2007. Potencial alelopático diferencial de cultivares de trigo (Triticum aestivum L.) chileno sobre ballica anual (Lolium rigidum) var. Wimmera. Idesia, 25 (2): 81-89.

Bensch, E.; Schalchli, H.; Jobet, C.; Seemann, P.; Fuentes, R. 2009. Potencial alelopático diferencial de trigo (Triticum aestivum $\mathrm{L}$.) chileno sobre algunas malezas asociadas al cultivo en el sur de Chile. Idesia, 27 (3): 77-88.

Bertholdsson, $\mathrm{N}$.

2004. Variation in allelopathic activity in spring wheat. In: Proceedings of Second European Allelopathy SymposiumAllelopathic: from understanding to application, p. 22. Pulawy, Poland, June 4, 2004.

Bowen, J.

1991. Las alelopatías en la producción agrícola. Revista Agricultura de las Américas, 40 (1): 8-11.

Bruce, S.; Christen, O.

2001. Phytitotoxity of wheat leachates and ferulic acid to germination and radicle elongation of canola. Proceedings of the $10^{\text {th }}$ Australian Agronomy Conference, Hobart.

Cheema, Z.; Khaliq, A.

2000. Use of sorghum allelopathic properties to control weeds in irrigated wheat in a semi arid region of Punjab. Agriculture, Ecosystems and Environment, 79: 105-112.

Chou, Ch.

1999. Roles of allelopathy in plant biodiversity and sustainable agriculture. Critical Reviews in Plant Sciences, 18: 609-636.

Chung, I.; Miller, D.

1995. Natural herbicide potential of alfalfa residue on selected weed species. Agronomy Journal, 87: 920-925.

Chung, I.; Ahn, J.; Yun, S.

2001. Assessment of allelopathic potential of barnyard grass (Echinochloa crus-galli) on rice (Oryza sativa L.) cultivars. Crop protection, 20: 921-928.

Copaja, S.; Niemeyer, H.; Wratten, S.

1991. Hydroxamic acids levels in Chilean an British wheat seedlings. Annals of Applied Biology, 118: 223-227.

Dilday, S.; Lin, J.; Yang, W.

1994. Identification of allelopathy in the USDA-ARS rice germplasm collection. Australian Journal of Experimental Agriculture, 34: 907-910.

Einhellig, F.

1995. Mechanism of action of allelohemicals in allelopathy. In: Inderjit, Ch.; Daskshini, K. and Einhelling, F. (eds). Allelopathy: Organisms, Processes, and Aplication. pp. 96-116.

Eskelsen, S.; Crabtree, G.

1995. The role of allelopathy in Buckwheat (Fagorypum sagittatum) inhibition of Canada thistle (Cirsum arvense). Weed Science, 43: 70-74.

Espinoza, N.

1996. Malezas presentes en Chile. Santiago, Chile. Editora Aníbal Pinto S.A. 219 p.

Evans, W.

1989. Trease and Evans' Pharmacognosy, $13^{\text {th }}$ edition. Bailliere Tindall, London, England, 832 p.

Fay, P.; Duke, W.

1977. An assessment of allelopathic potential in Avena germplasm. Weed Science, 25: 224-228.
Harborne, J.

1982. Introduction to Ecological Biochemistry, 2nd ed., Academic Press, New York, 278 p.

Inderjit, J.; Weston, L.

2000. Are laboratory bioassays for allelopathy suitable for prediction of field responses? Journal of Chemical Ecology, 26: 2111-2118.

Kogan, M.

1992. Malezas. Ecofisiología y estrategias de control. Santiago. Colección en agricultura. Facultad de Agronomía. Pontificia Universidad Católica de Chile, 402 p.

Laynez-Garsaball, J.; Méndez-Natera, J.

2006. Efectos de extractos acuosos del follaje del corocillo (Cyperus rotundus L.) sobre la germinación de semillas y el crecimiento de plántulas de ajonjolí (Sesamun indicum $\mathrm{L}$.) cv. arapatol s-15. Idesia (Chile), 24 (2): 61-75.

Mathiassen, S.; Mogensen, B.; Kudsk, P.

2004. Effects on weeds of soil-incorporated wheat. In: The Second European Allelopathy Symposium "Allelopathy from understanding to application”. Pulawy, Polonia, pp. 81-82.

Matthei, O.

1995. Manual de las malezas que crecen en Chile. Concepción. Chile $545 \mathrm{p}$.

Mogensen, B.; Mathiassen, S.; Krongaard, T.; Eljarrat, E.; Villagrasa, M.; Guillamón, M.; Taberner, A.; Barcelo, D.

2004. Qunatification of hidroxamic acid allelochemicals in wheat varieties grow under varying conditions. In: The Second European allelopathic Simposium "Allelopathy from understanding to application”. Pulawy, Polonia, pp. 50-53.

Olofsdotter, M.; Navarez, D.; Rebulanan, M.; Streibig, J.

1999. Weed-suppressing rice cultivars does allelopathy play role?. Weed research, 39: 441-454.

Ormeño, J.

1997. Aplicación de la alelopatía en el control de malezas: El caso del centeno (Secale cereale L.) en Chile. En: Producción de alimentos orgánicos. Seminario internacional. INIAQuilamapu, Chillán. Chile. 29-30 de octubre.

Ormeño, J.

1999. Manejo y control de malezas con plantas alelopáticas: Centeno. In: Agricultura orgánica. Céspedes, C. y Carvajal, P. Trama. Ministerio de Agricultura, INIA-Quilamapu, Chillán, Chile, pp. 121-137.

Pérez, F.; Ormeño, J.

1991. Difference in hidroxamic acid in roots and roots exudates of wheat (Triticum aestivum L.) and rye (Secale cereale $\mathrm{L}$.) possible role in allelopathy. Journal of Chemical Ecology, 17: 1037-1043.

Putnam, A.; Duke, V.

1974. Biological suppression of weeds: evidence for allelopathy in accessions of cucumber. Science, 185: 370-372.

Rice, E.

1984. Allelopathy, $2^{\text {nd }}$ Edition. New York, Academic Press. $422 \mathrm{p}$.

Rice, E.

1979. Allelopathy an update. Botanical Review (USA), 45: 15-109.

Tang, C.

1986. Continuous trapping techniques for the study of allelochemicals from higher plants. In: Putnam AR, Tang $\mathrm{C}-\mathrm{H}$ editors. The science of allelopathy. New York (USA) pp. 113-131. 
Wojcik, D.; Politycka, B.; Schneider, M.; Perkowski, J. 1990. Phenolic substances as allelopathic agents aresing during the degradation of rye (Secale cereale L.) tissues. Plant Soil 124: 143-147.

Wu, H.; Pratley, J.; Lemerle, D.; Haig, T.; Verbeek, B. 1998. Differential allelopathic potential among wheat accessions to annual ryegrass. In: Proceeding of the $9^{\text {th }}$ Australian Agronomy Conference (Eds. D.L. Michalk, J.E. Pratley). Wagga-Wagga, NSW, pp. 567-571.

Wu, H.; Pratley, J.; Lemerle, D.; Haig, T. 1999. Crop cultivars with allelopathic capability. Weed Research 39: 171-180.

Wu, H.; Pratley, J.; Lemerle, D.; Haig, T.

2000a. Laboratory screening for allelopathic potential of wheat (Triticum aestivum) accessions against annual ryegrass (Lolium rigidum). Australian Journal Research 51: 259-266.
Wu, H.; Haig, T.; Pratley, J.; Lemerle, D.

2000b. Distribution and exudation of allelochemicals in wheat Triticum aestivum. Journal of Chemical Ecology 26: 2141-2154.

Wu, H.; Pratley, J.; Lemerle, D.; Haig, T.

2001a. Allelopathy in wheat (Triticum aestivum). Annals of Applied Biology 139: 1-9.

Wu, H.; Pratley, J.; Lemerle, D.; Haig, T.

$2001 \mathrm{~b}$. Wheat allelopathic potential against a herbicideresistant biotype of annual ryegrass. In: Proceeding of the $10^{\text {th }}$ Australian Agronomy Conference. Hobart, Australia.

Wu, H.; Pratley, J.; Lemerle, D.; An, M.; Liu, D.

2007. Modern genomic approaches to improving allelopathic capability in wheat (Triticum aestivum L.). Allelopathy Journal, 19. 97-108.

Zheng, Y.; Zhao, Y.; Dong, F.; Liu, J.; Yao, J.; Hurle, K. 2007. Allelopathic effects of wheat extracts and DIMBOA on weeds. Allelopathy Journal 19: 171-178. 
